04

\title{
Эффективное описание влияния рефракции, применимое при интерпретации интерферометрических измерений плотности плазмы в малом токамаке
}

\author{
(C) А.В. Сидоров, ${ }^{1}$ О.Л. Круткин, ${ }^{1}$ А.Б. Алтухов, ${ }^{1}$ Е.О. Векшина, ${ }^{2}$ А.Д. Гурченко, ${ }^{1}$ Е.3. Гусаков, ${ }^{1}$ \\ М.Ю. Кантор, ${ }^{1}$ Д.В. Куприенко, ${ }^{1}$ С.А. Хитров ${ }^{1}$ \\ ${ }^{1}$ Физико-технический институт им. А.Ф. Иофффе РАН, \\ 194021 Санкт-Петербург, Россия \\ ${ }^{2}$ Санкт-Петербургский политехнический университет Петра Великого, \\ 195251 Санкт-Петербург, Россия \\ e-mail: sidorov@mail.ioffe.ru
}

Поступило в Редакцию 7 декабря 2021 г.

В окончательной редакции 12 января 2021 г.

Принята к публикации 14 января 2021 г.

Показано, что приближение прямолинейных лучей зондирования, обычно используемое при восстановлении профиля плотности плазмы из интерферометрических измерений, приводит к неприемлемо большим ошибкам в случае плотной плазмы. Предложен быстрый метод расчета интерферометрического сигнала, основанный на приближении геометрической оптики и учитывающий рефракцию зондирующей волны, который может быть использован при восстановлении профиля плотности плазмы. Адекватность метода подтверждена сравнением с результатами полноволнового расчета.

Ключевые слова: токамак, интерферометрия, полноволновой расчет, геометрическая оптика, рефракция.

DOI: $10.21883 / J T F .2022 .04 .52242 .310-21$

\section{Введение}

Микроволновая интерферометрия является широко распространенной диагностикой, рутинно используемой для измерения профиля электронной плотности на множестве токамаков. Как правило, диагностика основана на зондировании плазмы микроволновым пучком обыкновенной $(\mathrm{O})$ поляризации и измерении разности фаз между опорным сигналом и прошедшим через плазму излучением. Поскольку волновое число О-моды определяется плотностью плазмы, эта величина содержит информацию об интегральной плотности вдоль пути распространения пучка зондирования. На многих тороидальных установках зондирование плазмы выполняется в полоидальной плоскости, в вертикальном направлении, с использованием небольшого числа передающих и приемных антенн, расположенных друг напротив друга [1].

Для определения связи между характеристиками зондирующей электромагнитной волны и распределением плотности в плазме используют дисперсионное соотношение, которое для О-моды в декартовых координатах может быть записано как:

$$
k^{2}(x, y)=\frac{\omega^{2}}{c^{2}}\left(1-\frac{n(x, y)}{n_{c}}\right),
$$

где $k$ - величина волнового вектора зондирующей волны, $\omega$ - частота зондирования, $n-$ электронная плотность плазмы, а $n_{c}-$ критическая плотность, определяемая выражением $n_{c}=m_{e} \omega^{2} / 4 \pi e^{2}$, в котором $m_{e}$ - масса электрона, а $e$ - элементарный заряд.
Для нахождения набега фазы сигнала, прошедшего через двумерно неоднородную плазму, необходимо решить полноволновую задачу с учетом граничного условия на амплитуду падающей волны и диаграммы направленности приемной антенны. Тем не менее обычно предполагают, что этот фазовый набег связан с интегралом вдоль лучевой траектории зондирующей волны $L$, соединяющей зондирующий и приемный рупоры:

$$
\varphi=\frac{\omega}{c} \int_{L} \sqrt{1-\frac{n(x(l), y(l))}{n_{c}}} d l .
$$

При интерпретации данных измерений и восстановлении по ним профиля плотности зачастую предполагается, что зондирующий пучок не подвержен рефракции и набег его фазы может быть вычислен интегрированием волнового числа вдоль хорд, соединяющих приемные и передающие рупоры [2]. Это предположение, справедливое при условии, что плотность плазмы много меньше критического значения $\left(n \ll n_{c}\right)$, позволяет получить следующее выражение для недостающего (по сравнению с прохождением через вакуум) набега фазы зондирующей волны для всех хорд зондирования:

$$
\Delta \varphi_{i}=\frac{\omega}{c} \int_{L_{y}^{i}}\left(1-\sqrt{1-\frac{n\left(x_{i}, y\right)}{n_{c}}}\right) d y,
$$

где $L_{y}^{i}$ - хорда, соединяющая центры зондирующего и приемного рупоров. 
Из формулы (3) решается обратная задача о поиске распределения $n(x, y)$, удовлетворяющего измеренным фазам. Сложность данной задачи состоит в том, что такая система уравнений не имеет единственного решения. Существует бесконечное множество двумерных распределений плотности, которые при интегрировании по одной из координат дадут измеренное одномерное распределение фаз. Для преодоления этого препятствия на $n(x, y)$ накладываются дополнительные условия, связанные с предполагаемой формой магнитных поверхностей.

В разрядах с высокой плотностью, когда условие $\left(n \ll n_{c}\right)$ нарушается, рефракция оказывает существенное влияние на распространение зондирующего пучка и простая трактовка измеренного набега фазы, согласно формуле (3), неприменима [3]. В связи с этим необходимо разработать метод определения связи измеряемого экспериментально с помощью интерферометрической схемы набега фазы и характеристик профиля плотности плазмы в присутствии рефракции. В частности, такая задача возникла в процессе исследования разрядов высокой плотности на токамаке ФТ-2 [4]. Настоящая работа посвящена разработке метода учета рефракции зондирующего пучка для процедуры восстановления профиля плотности плазмы из измерений микроволнового интерферометра на токамаке. Необходимость и эффективность разработанных подходов для интерпретации данных измерений в разрядах высокой плотности демонстрируется на примере токамака ФТ-2.

\section{1. Интерферометрическая диагностика}

\section{1. Основные технические детали}

Представим основные технические детали интерферометрической диагностики на примере системы, эксплуатируемой на токамаке ФТ-2. ФТ-2 - небольшая исследовательская установка с большим радиусом $55 \mathrm{~cm}$ и малым радиусом $8 \mathrm{~cm}$ [5]. Токамак оснащен микроволновым интерферометром, зондирующим плазму на частоте $135 \mathrm{GHz}$ вдоль семи вертикальных хорд, лежащих в одном полоидальном сечении. Критическая плотность, соответствующая частоте зондирования, составляет $2.3 \cdot 10^{14} \mathrm{~cm}^{-3}$. Схема геометрии интерферометра ФТ-2 изображена на рис. 1.

До недавнего времени для восстановления распределения плотности из интерферометрических измерений на токамаке ФТ-2 использовался алгоритм, основанный на алгоритме $[6,7]$, в предположении, что магнитные поверхности в плазме - это вложенные друг в друга окружности, центры которых смещены по большому радиусу (шафрановский сдвиг).

Алгоритм [6] был доработан для восстановления несимметричных по большому радиусу профилей плотности с большими градиентами за счет более точного определения радиусов восстанавливаемых магнитных

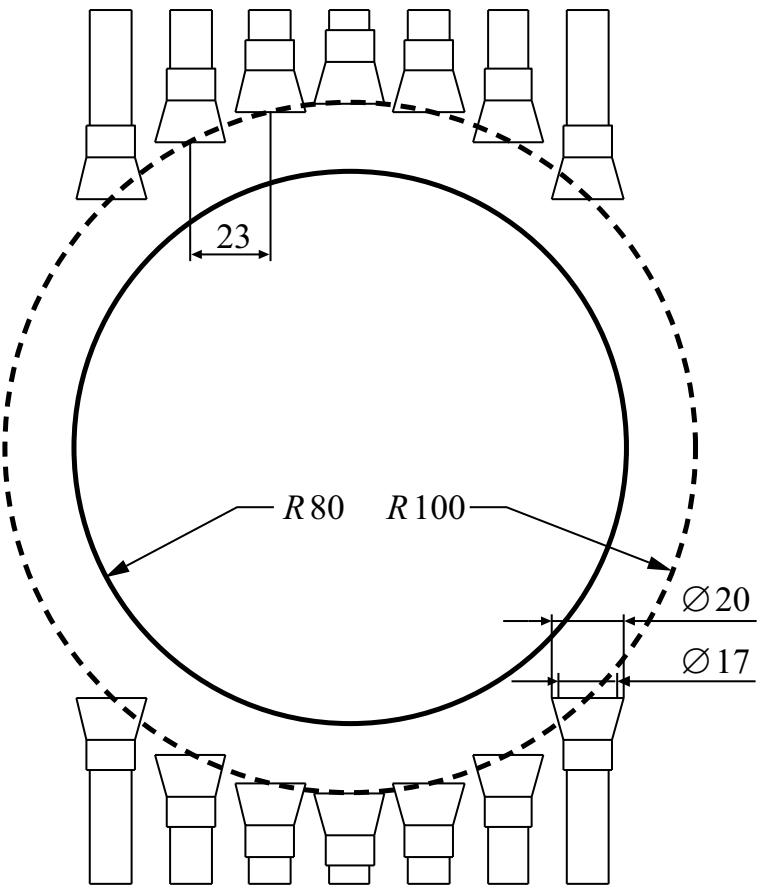

Рис. 1. Схема расположения интерферометрических антенн на токамаке ФТ-2.

поверхностей. Для устойчивости обработки по отношению к погрешностям измерений измеренные фазы интерполировались с помощью сплайна с задаваемой степенью жесткости. Разработанный алгоритм обеспечивает надежное восстановление локальной плотности и магнитной конфигурации в плазме со средней плотностью до $4 \cdot 10^{13} \mathrm{~cm}^{-3}$. Однако электронная плотность в экспериментах [4] может превышать $10^{14} \mathrm{~cm}^{-3}$, что всего в два раза ниже критической плотности, и, следовательно, условие $n \ll n_{c}$ не выполняется. При таких высоких плотностях необходимо учитывать рефракцию пучка и, как будет показано далее, его конечную ширину, а значит использование в полном объеме существующей процедуры реконструкции профиля плотности приведет к неверным результатам.

\section{2. Полноволновой расчет}

Для того чтобы получить максимально полное и точное представление о характере распространения микроволноволнового излучения в интерферометре токамака ФТ-2, было проведено полноволновое моделирование.

Моделирование было выполнено с помощью кода CUWA [8], основанного на вычислении временно́й эволюции электрического и магнитных полей с помощью метода конечных разностей. Для расчета были использованы двумерная версия кода, исключающая тороидальные эффекты, и декартова система координат. В качестве граничного условия для зондирующего излучения в полноволновом расчете был использован гауссов пучок с параметрами максимально близкими к 

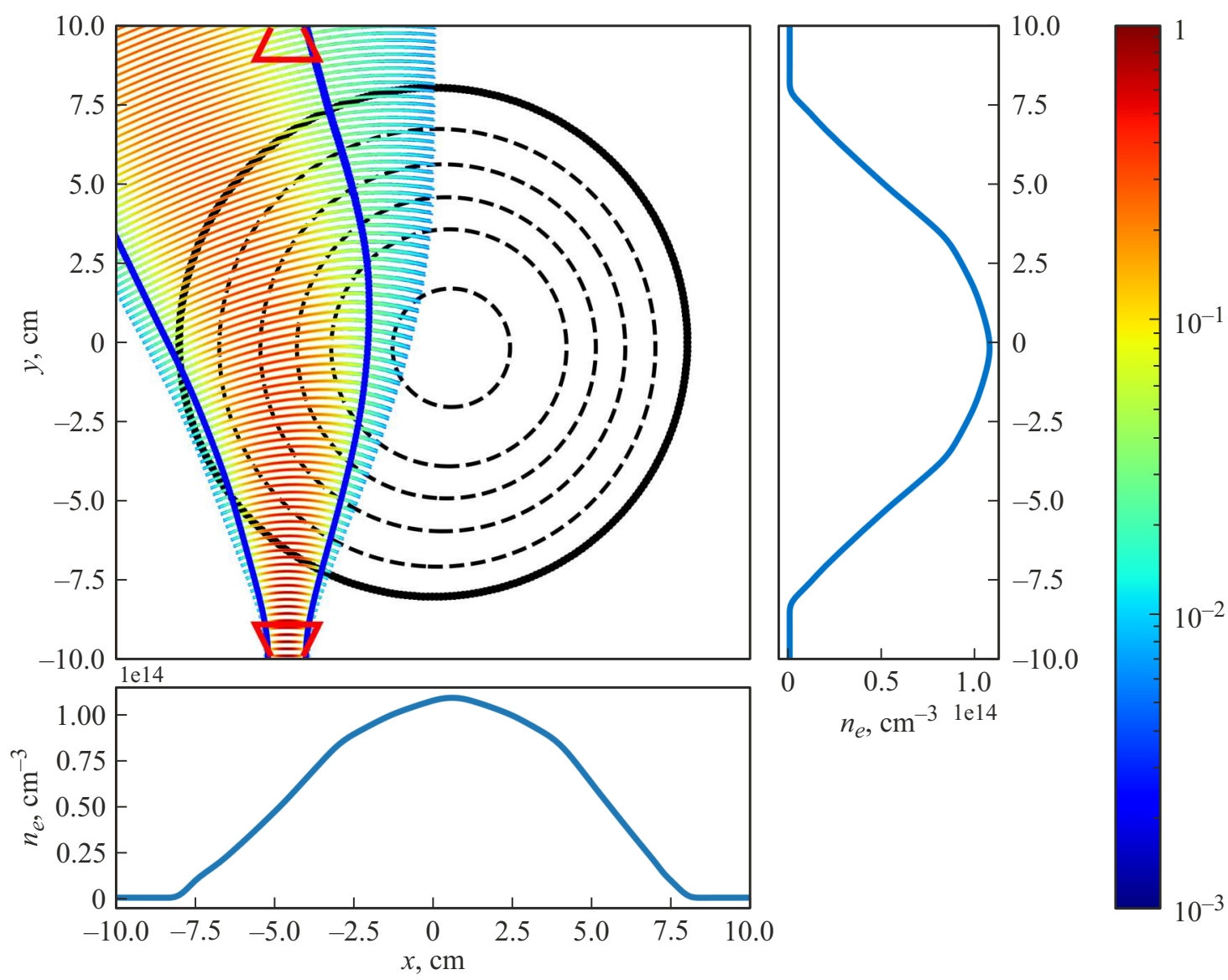

Рис. 2. Абсолютная величина действительной части электрического поля для положения антенны $-4.6 \mathrm{~cm}$ (в произвольных единицах) в области, где амплитуда поля превышает $1 \%$ от максимального значения. Черные штриховые линии соответствуют контурам постоянной плотности. Синие сплошные линии (в онлайн версии) соответствуют контурам уровня 5\% от максимальной амплитуды поля. Красным (в онлайн версии) отображены рупоры излучающей и принимающей антенн. Приведены профили плотности по экваториальной и центральной хорде.

экспериментальным пучкам. Последние, в свою очередь, были получены при помощи моделировании излучения волнового тракта (прямоугольный волновод, переход на круглый, вакуумная развязка, диэлектрическая развязка, круглый волновод и рупор).

Параметр, определяющий ширину пучка (по уровню амплитуды $1 / e$ в перетяжке), оказался равен $\rho_{0}=3.5 \mathrm{~mm}$, а его перетяжка расположена на расстоянии $f=1 \mathrm{~cm}$ позади раскрыва рупора антенны.

На рис. 2 приведен пример расчета зондирующего электрического поля в плазме с помощью полноволнового кода CUWA [8] для положения антенны, сдвинутого в горизонтальном направлении относительно оси камеры в направлении оси симметрии токамака на $-4.6 \mathrm{~cm}$ (рис. 1). Профиль плотности, соответствующий этому расчету (и основанный на экспериментальных измерениях), показан на рис. 2 по бокам от основного рисунка.

Формула (3) применима, когда смещение и разворот поверхности постоянной фазы в зондирующем пучке изза рефракции невелики, так что набег фазы, соответствующий разным лучам, пришедшим в апертуру антенны, отличается слабо и близок к посчитанному по прямой, соединяющей центры зондирующего и приемного рупоров. На рис. 2 видно, что при плотностях плазмы на оси разряда масштаба $10^{14} \mathrm{~cm}^{-3}$ как смещение центрального луча, так и разворот фазового фронта существенны, так что приемный рупор регистрирует сигнал, создаваемый периферией зондирующего пучка. Для корректного вычисления фазы этого сигнала необходимо рассчитать полную картину зондирующего поля на принимающей антенне с учетом рефракции.

Таким образом, для учета полной физической картины необходимо учитывать двумерное распределение поля, а также пространственные характеристики рупора приемной антенны.

Для конкретной плотности плазмы $n_{0}(x, y)$ полноволновой расчет позволяет определить набег фазы зондирующей волны, но лишь с точностью до целого числа $2 \pi$. Это связано с методом определения фазы в расчете. Сигнал на принимающей антенне $S$ определяется (в соответствии с теоремой взаимности) путем интегрирования поля зондирующей волны с виртуальным сигналом 
принимающей антенны на линии, параллельной оси $x$, на рис. 2:

$$
\begin{gathered}
S \propto \int_{-10}^{10} E_{z}\left(x, y_{a}\right) E_{a}\left(x, y_{a}\right) d x, \\
E_{a}\left(x, y_{a}\right)=E_{0 z} \sqrt{\frac{\rho_{0}}{\rho_{a}}} \exp \left(-\frac{\left(x-x_{0}\right)^{2}}{\rho_{a}^{2}}\right) \\
\times \exp \left(-i\left[k\left(y_{a}-y_{0}\right)+k \frac{\left(x-x_{0}\right)^{2}}{2 R_{a} \lambda}+\Psi_{a}\right]\right),
\end{gathered}
$$

где (4b) соответствует виртуальному сигналу антенны, где $\lambda-$ длина волны, $k=\frac{2 \pi N}{\lambda}-$ волновой вектор в среде с коэффициентом преломления $N, y_{r}=\frac{\pi \rho_{0}^{2} N}{\lambda}-$ длина Рэлея в среде с коэффициентом преломления $N, E_{0 z}$ - обеспечивает единичное значение мощности, излучаемой приемной антенной, когда она работает на излучение, $\rho_{0}-$ полуширина виртуального пучка в его перетяжке - расстояние от оси пучка, на котором амплитуда поля падает в $e$ раз, $\rho_{a}=\rho_{0} \sqrt{1+\frac{\left(y_{a}-y_{0}\right)^{2}}{y_{r}^{2}}}-$ полуширина виртуального пучка на расстоянии $y_{0}-y_{a}$ от перетяжки по оси пучка, $R_{a}=\left(y_{a}-y_{0}\right)\left(1+\frac{y_{r}^{2}}{\left(y_{a}-y_{0}\right)^{2}}\right)-$ радиус кривизны волнового фронта, $\Psi_{a}=\arctan \frac{y_{a}-y_{0}}{y_{r}} / 2-$ фаза Гуи, $x_{0}$ и $y_{0}$ - координаты оси и перетяжки виртуального пучка.

Формула (4b) соответствует двумерному гауссову пучку, поскольку проведенное численное моделирование также было выполнено на двумерной сетке.

Выбор линии $y=y_{a}$ для записи был обусловлен положением интерферометрических антенн в эксперименте $\left(y_{a}-y_{0}\right.$ при этом оказывается положительной величиной $f=1 \mathrm{~cm}$, соответствующей расстоянию от раскрыва рупора антенны до перетяжки соответствующего ей гауссова пучка). Фаза получившегося комплексного сигнала может быть определена с точностью до целого числа периода синуса, т. е. в пределах $[0,2 \pi]$. Оценку опущенного при этом целого числа периодов может дать фазовый интеграл вдоль центрального луча, испущенного из зондирующей антены. Однако в случае сильного смещения и разворота зондирующего пучка в эту оценку может вкрасться ошибка, превосходящая $2 \pi$. Точное значение набега фазы может быть восстановлено путем проведения набора расчетов с плотностью $a n_{0}(x, y)$, где $a$ - константа, меняющаяся в пределах $[0,1]$. В таком случае набег фазы будет постепенно нарастать и „обнуляться“ при достижении 2л. Учитывая количество таких „обнулений“, можно определить полное значение набега фазы. Такой подход воспроизводит экспериментальные измерения и позволяет определить набег фазы, несмотря на разворот и смещение фазового фронта.

\section{2. Восстановление плотности в случаях, когда необходимо учитывать рефракцию}

\section{1. Общее описание принципа восстановления}

Поскольку традиционный подход, основанный на формуле (3) [6,7], неприменим в условиях токамака ФТ-2 для разрядов с высокой плотностью необходимо разработать альтернативный метод определения профиля электронной плотности плазмы. Для решения этой задачи перспективным представляется использование итерационных численных методов.

Предполагаемая процедура восстановления состоит в подборе распределения электронной плотности, которое при расчете с учетом рефракции зондирующего пучка дает набеги фаз, совпадающие с экспериментальными измерениями. Так как „угадать“ распределение затруднительно, то его определение должно происходить путем постепенной корректировки некоего исходного профиля плотности и магнитной конфигурации до момента удовлетворительного, в пределах погрешностей измерений, совпадения экспериментальных и рассчитанных фазовых набегов. Такая процедура требует многократного проведения расчета фазовых набегов с учетом рефракции зондирующей волны. Однако многократное проведение полноволновых расчетов требует значительных вычислительных ресурсов и делает рутинное использование такой итерационной процедуры затруднительной. В связи с этим в рамках настоящей работы был разработан альтернативный быстрый метод определения фазы зондирующего сигнала из профиля плотности $n_{0}(x, y)$, основанный на методе геометрической оптики (ГО).

Разработка процедуры восстановления, в том числе принципов выбора исходного распределения плотности и методов ее корректировки является предметом будущей работы.

\section{2. Использование приближения геометрической оптики для определения фазы при интерферометрических измерениях}

Метод ГО позволяет рассчитать изменения фазы и амплитуды волны в пучке вдоль лучевой траектории, получаемой при интегрировании системы уравнений:

$$
\begin{gathered}
\frac{\partial x}{\partial l}=\frac{k_{x}}{k}, \quad \frac{\partial y}{\partial l}=\frac{k_{y}}{k}, \quad \frac{\partial k_{x}}{\partial l}=-\frac{1}{2 n_{c}} \frac{\omega^{2}}{c^{2} k} \frac{\partial n}{\partial x}, \\
\frac{\partial k_{y}}{\partial l}=-\frac{1}{2 n_{c}} \frac{\omega^{2}}{c^{2} k} \frac{\partial n}{\partial y} .
\end{gathered}
$$

Эта система соответствует прямоугольной системе координат, приведенной на рис. 2. $x, y-$ координаты луча, $k_{x}, k_{y}$ - компоненты его волнового вектора длиной $k, l$ - независимая переменная, имеющая смысл 
длины луча. Интегрирование этой системы уравнений позволяет получить траекторию зондирующего луча с учетом рефракции.

\section{3. Алгоритм работы, выбор корректного начального фронта, пересчет амплитуды}

Согласно [9], алгоритм работы метода состоит из ряда шагов:

1. Перпендикулярно волновому фронту, излучаемому зондирующей антенной, генерируется набор лучей, соответствующих зондирующему пучку и описываемых системой уравнений (5). Каждому лучу соответствуют стартовые координаты и волновой вектор, а также амплитуда и фаза. Выбор стартовых параметров лучей оказывает существенное влияние на итоговое значение фазы. Поскольку распространение лучей должно описывать расхождение пучка, следует выбирать лучи, направленные именно перпендикулярно волновому фронту зондирующего пучка. Для описания антенн интерферометра на токамаке ФТ-2 использовался гауссов пучок и область перетяжки, где приближение ГО нарушается, оказалась внутри антенны, всего в одном сантиметре от ее раскрыва. Поэтому для расчета лучевых траекторий в качестве начального фронта был выбран волновой фронт, находящийся вне антенны и отстоящий от перетяжки пучка на $3 \mathrm{~cm}$ ( $2 \mathrm{~cm}$ от раскрыва рупора).

2. Чтобы найти лучевую траекторию в соответствии с параметрами луча и системой уравнений (5), рассчитывается дискретный „шаг“ вдоль ее длины. Каждому лучу присваиваются скорректированные значения координат и волнового числа. Набег фазы вычисляется как $k_{x} \Delta x+k_{y} \Delta y$, где $\Delta x, \Delta y-$ приращение $x$ и $y$ за этот шаг. Амплитуда пучка корректируется в соответствии с увеличением расстояния между лучами, для сохранения постоянным потока энергии зондирующего излучения. Этот этап выполняется множество раз, пока лучевая траектория не достигнет области принимающей антенны.

3. Когда все лучи пересекают линию, соответствующую рупору принимающей антенны, значения фаз и амплитуд на лучах интерполируются на регулярную сетку и дают значения поля зондирующей волны:

$$
E_{z}\left(x_{j}, y_{a}\right)=A_{j}\left(y_{a}\right) \exp \left(i\left[\int_{0}^{l_{a}} k\left(x_{j}(l), y_{j}(l)\right) d l\right]\right) .
$$

Эти значения интегрируются с виртуальным сигналом принимающей антенны по аналогии с полноволновым расчетом и формулой $(4 \mathrm{a})$.

Значения набега фазы сигнала интерферометра при этом, как и в полноволновом расчете, получаются путем проведения серии расчетов с постепенным наращиванием плотности от малых значений при неизменной форме профиля. Чтобы подтвердить предложенный подход, можно сравнивать как вычисленное значение фазы, так и распределение поля зондирующей волны на приемной антенне, восстановленное в приближении геометрической оптики, со значениями, полученными при полноволновом моделировании.

\section{4. Проверка предложенного метода с помощью полноволнового расчета}

Для подтверждения результатов, полученных с помощью метода ГО, было использовано полноволновое моделирование с помощью кода CUWA. Первым шагом сравнения стало проведение расчета в вакууме для проверки описания поведения гауссова пучка. Пример сравнения вещественной и мнимой частей электрического поля в плоскости принимающей антенны приведен на рис. 3.

Рисунок соответствует вакуумному зондированию из наиболее удаленных приемной и излучающей антенн на оси разряда (расстояние между ними $20 \mathrm{~cm}$ согласно рис. 1). Из рисунка видно, что метод ГО корректно описывает поведение зондирующей волны в интервале гораздо шире геометрической области, ограниченной принимающей антенной. В вакууме мы можем расчитать поле аналитически - поле гауссова пучка (см. описание к $(4 b))$. Это поле приведено с помощь черной штриховой кривой. Голубая и оранжевая кривые (в онлайн версии) отвечают старту ГО лучей соответственно в 2 и $6 \mathrm{~cm}$ от рупора и иллюстрируют соображение приведенные в разд. 2.3, п. 1. Чем дальше от перетяжки стартовые координаты лучей, тем точнее расчеты с помощь метода ГО совпадают с аналитической формулой (4b) и полноволновым расчетом. К сожалению, задавать начальные координаты дальше $2 \mathrm{~cm}$ от раскрыва рупора невозможно, так как там находится граница плазменного шнура (срез диафрагмы). Тем не менее и при этом стартовом положении соответствие результатов ГО и полноволновых расчетов вакуумного пучка вполне удовлетворительное, что позволяет рассчитывать на корректный расчет фазы сигнала интерферометра с помощью метода ГО. Следующим этапом сравнения метода ГО и полноволнового кода стал расчет в присутствии плазмы при наличии рефракции. Примером такого расчета для профиля плотности, приведенного на рис. 2, и положения антенны $-4.6 \mathrm{~cm}$ в горизонтальном направлении от оси камеры токамака приведен на рис. 4.

При наличии существенной рефракции (максимум поля существенно сдвинут от значения $-4.6 \mathrm{~cm}$, соответствующего положению принимающей и излучающей антенн), которая делает формулу (3) неприменимой, метод ГО хорошо описывает поле в области приемного рупора и, следовательно, позволяет рассчитать корректное значение набега фазы. В ходе сравнения результатов была обнаружена ситуация, когда использование метода ГО дает не полностью корректный результат. Поля для этого случая приведены на рис. 5 и соответствуют положению антенн $+6.9 \mathrm{~cm}$ относительно центра камеры. 

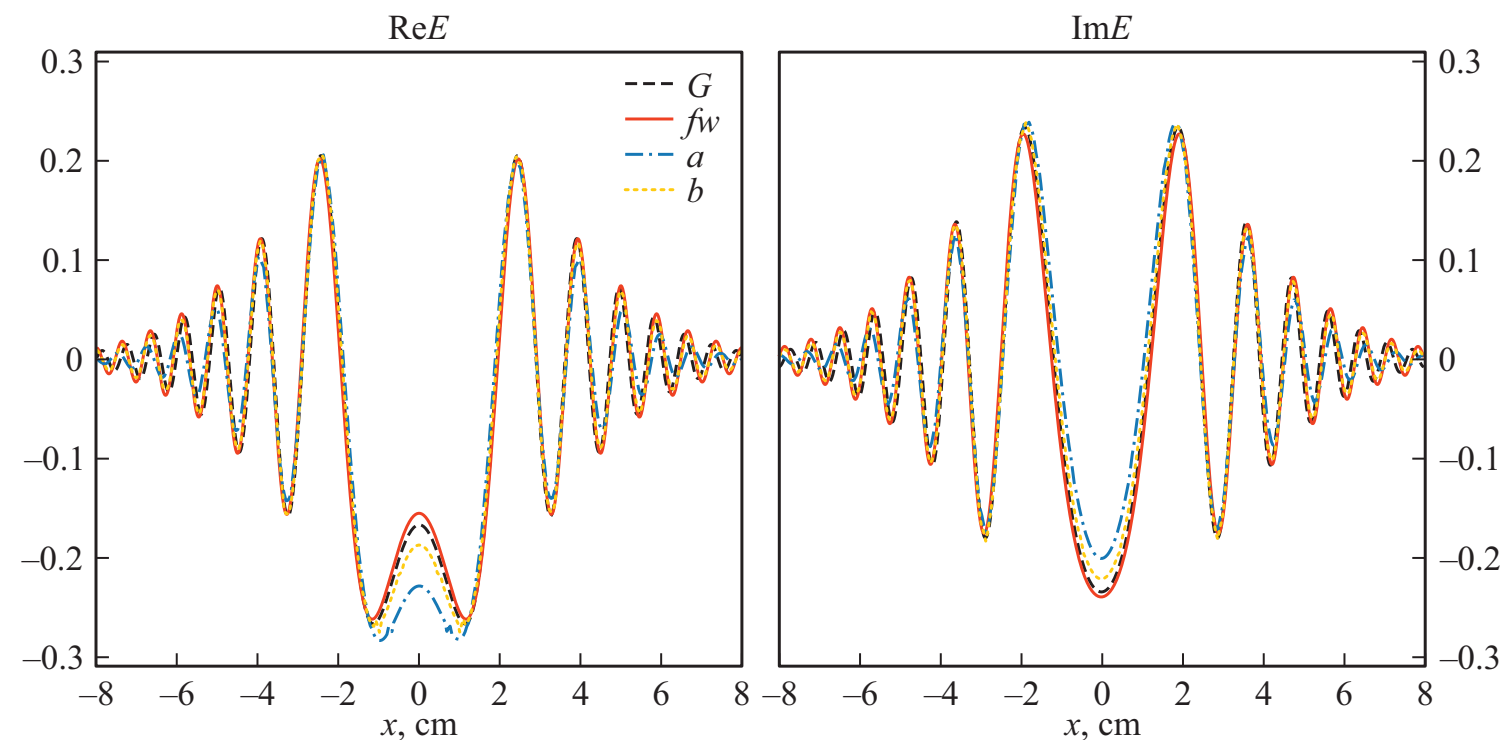

Рис. 3. Вещественная и мнимая части электрического поля, посчитанные полноволновым кодом (красная сплошная линия $f w$ ), и методом ГО (линии $a$ - старт лучей в $2 \mathrm{~cm}$ от рупора и $b-$ старт лучей в $6 \mathrm{~cm}$ от рупора) при зондировании в отсутствие плазмы. Черная штриховая линия $G$ соответствует аналитическому выражения для двумерного гауссова пучка (4b).
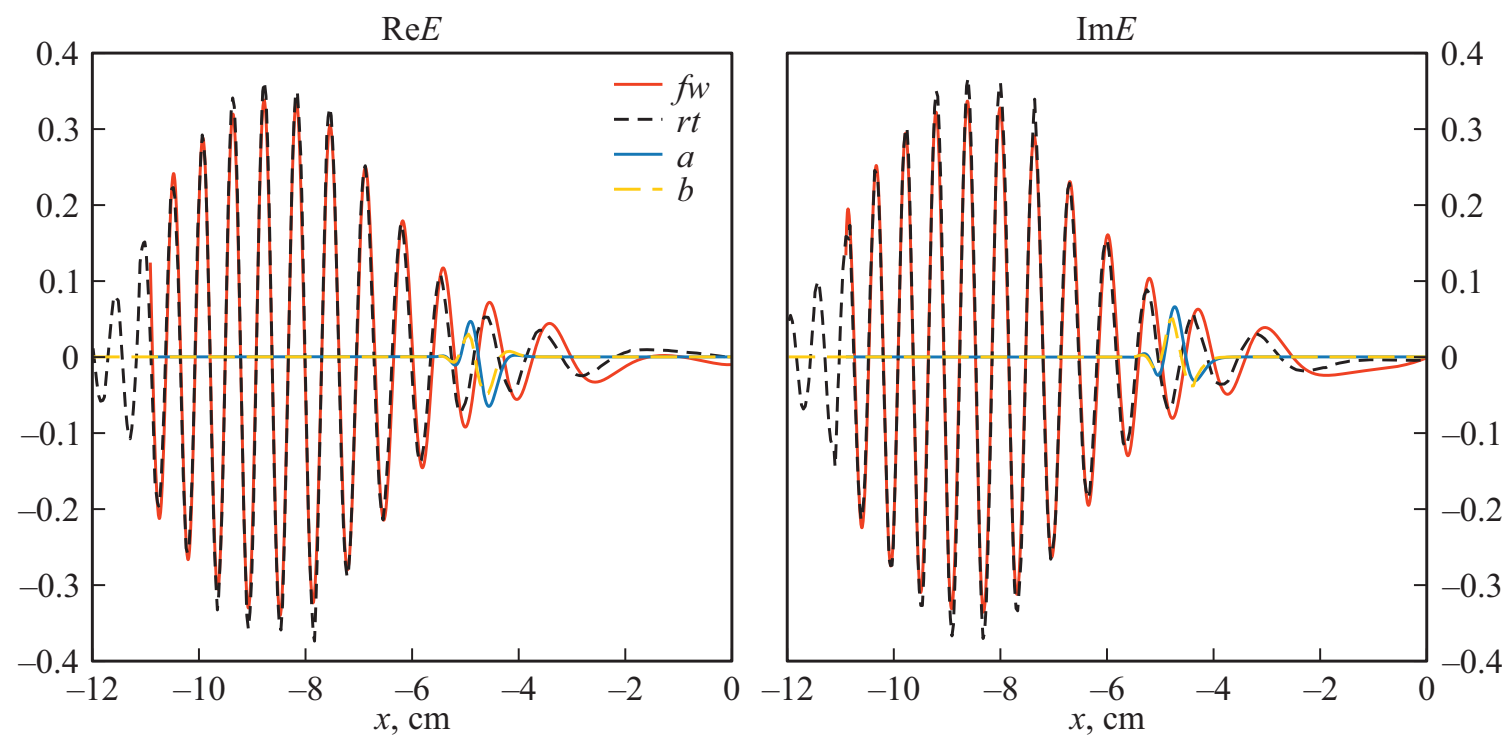

Рис. 4. Вещественная и мнимая части электрического поля, посчитанные полноволновым кодом (красная линия $f w)$ и методом ГО (черная штриховая линия $r t$ ). Толстая сплошная голубая (a) (полноволновой код) и оранжевая штриховая $(b)$ (ГО) линии соответствуют произведению зондирующего поля и диаграммы приемной антенны, определяющей принимаемый сигнал (согласно формуле (4a)). Положение антенны $-4.6 \mathrm{~cm}$.

Трудности описания поля в этой ситуации связаны с тем, что сильный градиент плотности в области зондирования приводит к образованию каустики: лучи в расчете начинают пересекаться и определение амплитуды волны на них и интерполяция сигнала в пространстве становятся затруднительны. Иллюстрацией этого явления является рис. 6 , на котором приведены рассчитанные лучи для случаев, приведенных на рис. 4 и 5.

Наличие каустики вблизи антенны делает быстрый метод подсчета фазы неприменимым, и приводит в необходимости полноволнового расчета. К счастью, обнаружение такого случая по траекториям лучей не составляет труда. Более того, в случае, приведенном на рис. 6, каустика удалена от приемной антенны и не оказывает существенного влияния на интерферометрический сигнал.

Помимо сравнения полей на антенне было проведено сравнение фаз. На рис. 7 приведены фазы, посчитанные различными способами для описанных ранее случаев, а также двух других положений антенны. 

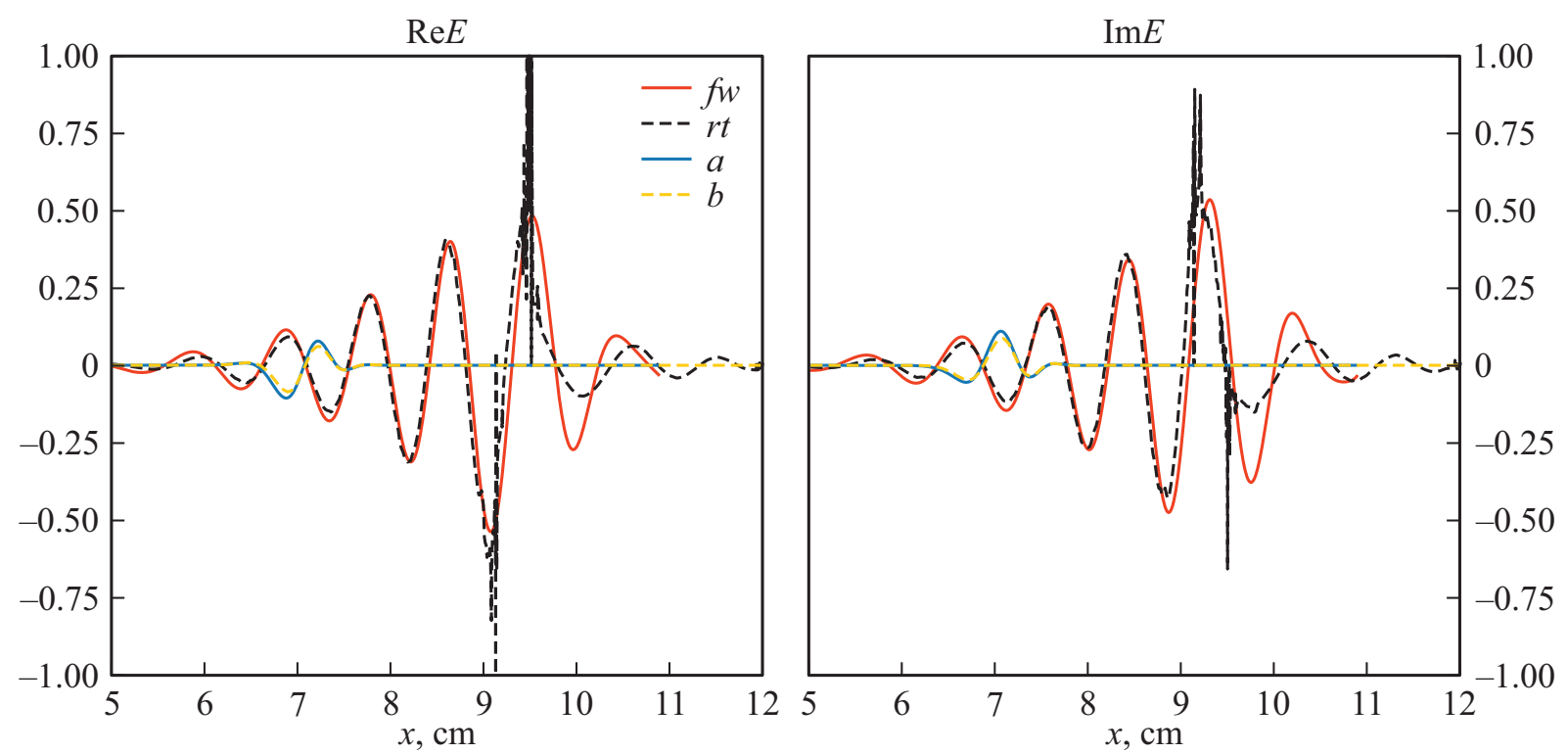

Рис. 5. Вещественная и мнимая части электрического поля, посчитанные полноволновым кодом (красная линия $f w)$ и методом ГО (черная штриховая $r t$ ). Толстая сплошная голубая $(a)$ (полноволновой код) и оранжевая штриховая $(b)($ ГО) линии соответствуют произведению зондирующего поля и диаграммы приемной антенны, определяющей принимаемый сигнал (согласно формуле (4а)). Положение антенны $+6.9 \mathrm{~cm}$.

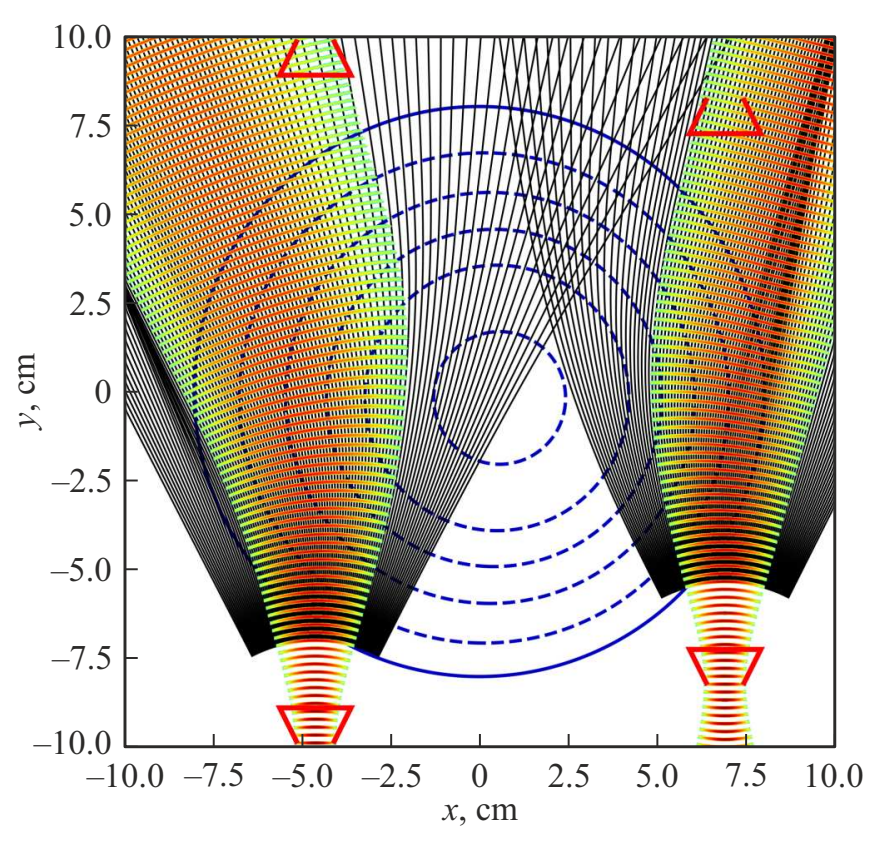

Рис. 6. Траектории лучей (черные тонкие линии), рассчитанные с помощью метода ГО для положения антенн -4.6 и $+6.9 \mathrm{~cm}$.

Из рис. 7 видно, что метод ГО дает значения фазы ближе к полноволновым по сравнению с традиционными упрощенными методами. Более того, даже для $a=0.5$, когда плотность уменьшена вдвое и становится существенно меньше критической, различие фаз между полноволновым расчетом и упрощенной формулой (3) все еще оказывается значительным для некоторых хорд.
Такой результат говорит о том, что учет фазового набега лишь вдоль линии, соединяющей центры передающего и принимающего рупоров, даже для низких плотностей может быть некорректным и использование более полного метода определения фазы представляется необходимым. Для случая, представленного на рис. $7, b$, все три результата совпадают за счет отсутствия существенного градиента плотности перпендикулярно зондирующему лучу при центральном зондировании.

\section{5. Преимущество метода ГО в скорости по сравнению с полноволновым расчетом}

Было также проведено сопоставление времени расчета полноволновым методом и методом ГО. Расчет CUWA для описанного в данном разделе случая был выполнен на сетке $2200 \times 2200$ точек. CUWA - код, проводящий вычисления на графических картах с использованием технологии CUDA. Расчет для профиля плотности с рис. 2 занял около $140 \mathrm{~s}$ на видеокарте NVidia GeForce GTX 1060 объемом 6 Gb. Эквивалентный расчет методом трассировки лучей в среде Python на том же устройстве (с процессором Intel i7-8540Н и $16 \mathrm{~Gb}$ оперативной памяти) занял около $1 \mathrm{~s}$. При этом для „быстрого“ расчета был использован 501 зондирующий луч. С учетом того что для получения полного значения фазы необходимо провести серию расчетов, включающую десятки отдельных вычислений, преимущество в скорости оказывается существенным. 

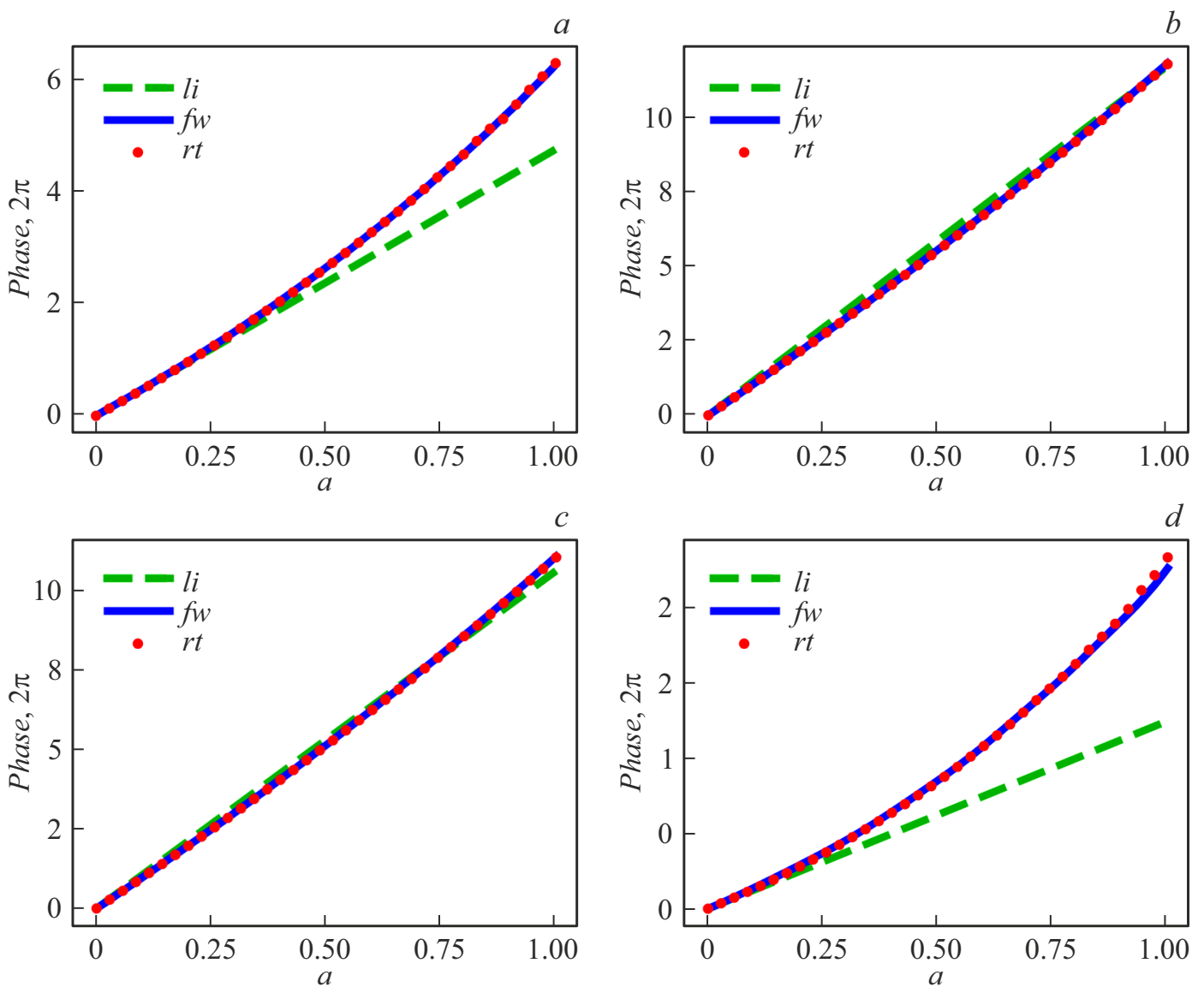

Рис. 7. Набег фазы, рассчитанный различными методам для положения антенн $a-4.6 \mathrm{~cm}, b+0 \mathrm{~cm}, c+2.3 \mathrm{~cm}$ и $d+6.9 \mathrm{~cm}$. По оси абсцисс отложено значение масштабирующего коэффициента $a$ в соответствии с описанием в разд. 1. По оси ординат отложен набег фазы в единицах $2 \pi$, посчитанный полноволновым методом (синяя линия $f w$ ), быстрым методом ГО (красные круги $r t$ ), в пренебрежении рефракцией согласно формуле (3) (зеленая пунктирная линия $l i$ ).

\section{6. Ограничения и применение „быстрого метода“}

Одно из существенных ограничений „быстрого метода“ уже было продемонстрировано в предыдущем разделе настоящей работы. Наличие каустик делает применение метода некорректным, так как „перемешивание“ амплитуды и фазы различных лучей в расчете затрудняет интерполяцию поля на принимающей антенне. Тем не менее по виду фазовой кривой и картины лучей (рис. 6) зачастую можно определить оказывает ли наличие каустик влияние на результат расчета интерферометрического сигнала. Однако, помимо этого, еще одно ограничение связано с самим методом ГО. Этот метод является приближенным и не учитывает дифракцию волнового пучка. В случае гауссова пучка, который описывает антенны токамака ФТ-2, зондирующий пучок имеет длину волны $\lambda=2.2 \mathrm{~mm}$ и полуширину в перетяжке $\rho_{0}=3.5 \mathrm{~mm}$. Длина Рэлея при этом оказывается $y_{r}=17.5 \mathrm{~mm}$ (см. описание к формуле $(4 \mathrm{~b})$ ), что позволяет использовать приближение ГО при запуске зондирующих лучей с поверхности, отстоящей от перетяжки на $3 \mathrm{~cm}$. Применимость метода ГО подтверждает также рис. 3. В общем случае необходимо проверять применимость приближения геометрической оптики для конкретных параметров зондирования.

Вторым аналогичным ограничением является необходимость правильного выбора стартовых параметров лучей. Если волновой фронт, с которого выполняется запуск лучей, находится слишком близко к фокусной плоскости зондирующего пучка, где приближение ГО нарушается, его кривизна не будет соответствовать дальнейшему расхождению лучей. В случае ФТ-2 этот эффект оказался не слишком значительным. Тем не менее для его устранения в расчетах для восстановления плотности был использован фронт, отстоящий от фокусной плоскости зондирующего пучка на $3 \mathrm{~cm}$.

Итоговое исследование метода ГО, представленное в предыдущих разделах, было выполнено на профиле плотности, представленном на рис. 2. Этот профиль плотности соответствует разряду ФТ-2 с высокой плотностью. Для иллюстрации близости выбранного профиля плотности экспериментальному на рис. 8 приведены набеги фазы, измеренные в эксперименте на ФТ-2, вместе с результатами моделирования. Высокая погрешность в измерении на $+4.6 \mathrm{~cm}$ связана с техни- 


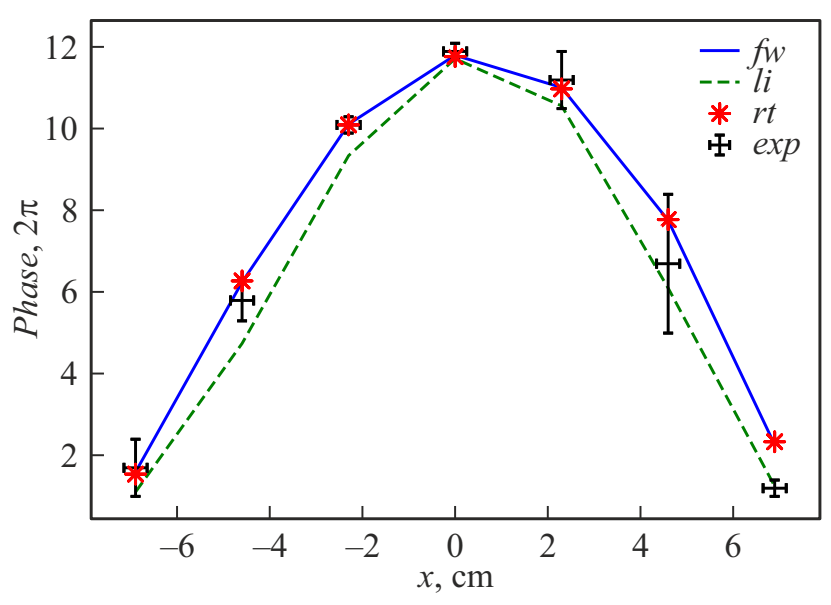

Рис. 8. Значение набега фазы в зависимости от положения антенны, измеренное в эксперименте (черные пределы погрешностей ехр), рассчитанное в полноволновом расчете (синяя сплошная линия $f w$ ), с помощью метода ГО (красные ${ }^{*} r t$ ) и путем интегрирования вдоль прямых линий (формула (3)) (зеленая штриховая линия $l i$ ).

ческими трудностями, возникшими в ВЧ аппаратуре во время эксперимента. Хотя фазы, полученные с помощью полноволнового моделирования, не дают полного совпадения с экспериментальными, они достаточно близки к ним, чтобы сделать вывод о реалистичности восстановленного профиля, выбранного для моделирования.

Что касается применения „быстрого“ метода ГО, он может быть использован как в процедуре восстановления профиля плотности, описанной в начале этого раздела, так и для проверки применимости традиционной интерпретации интерферометрических измерений. Вычисляя набеги фаз по восстановленному профилю, можно проверить их совпадение с экспериментально определенными фазами.

\section{Заключение}

Был разработан численный метод, использующий приближение ГО, для определения набега интерферометрической фазы. Метод позволяет учесть эффекты рефракции, которыми зачастую пренебрегают при анализе интерферометрических измерений. При этом скорость расчета оказывается существенно больше скорости полноволновых вычислений. Новый метод может быть интегрирован в существующие процедуры восстановления профиля плотности и стать основой для новых процедур, а также может быть использован для проверки соответствия итоговых профилей плотности экспериментальным измерениям.

\section{Финансирование работы}

Разработка геометрико-оптического подхода к расчету сигнала интерферометра, представленное в разд. 2.1, 2.2 и 2.3, поддержана в рамках государственного задания ФТИ им. А.Ф. Иоффе 0040-2019-0023, в то время как полноволновая его валидация, описанная в разд. 2.4 и 2.5, поддержана в рамках государственного задания ФТИ им. А.Ф. Иоффе 0034-2021-0001.

\section{Конфликт интересов}

Авторы заявляют, что у них нет конфликта интересов.

\section{Список литературы}

[1] В.Е. Голант. Сверхвысокочастотные методы исследования плазмы (Гл. ред. физматлит, Наука, М., 1968)

[2] В.Е. Голант. Методы диагностики, основанные на взаимодействии электромагнитного излучения с плазмой в кн. Основы бизики плазмы. Т. 2. Под ред. А.А. Галеев, Р. Судан (Энергоатомиздат, М., 1984), гл. VII, с. 534.

[3] Equipe TFR. Nucl. Fusion, 18, 647 (1978). DOI: $10.1088 / 0029-5515 / 18 / 5 / 004$

[4] Д.В. Куприенко, А.Б. Алтухов, А.Д. Гурченко, Е.З. Гусаков, Л.А. Есипов, О.А. Каледина, Н.В. Тропин, С.И. Лашкул, Г.А. Трошин, С.В. Шаталин. Физика плазмы, 45 (12), 1103 (2019). DOI: 10.1134/S0367292119120047

[5] N.N. Bakharev, G.I. Abdullina, V.I. Afanasyev, A.B. Altukhov, L.G. Askinazi, N.A. Babinov, A.N. Bazhenov, A.A. Belokurov, M.D. Blekhshtein, E.N. Bondarchuk. Nuclear Pasion, 59 (11), 112022 (2019). DOI: 10.1088/1741-4326/AB22DC

[6] N. Gottardi. J. Appl. Phys., 50 (4), 2647 (1979). DOI: $10.1063 / 1.326221$

[7] N. Gottardi. J. Appl. Phys., 50, 2647 (1979). DOI: $10.1063 / 1.328402$

[8] P. Aleynikov, N.B. Marushchenko. Comp. Phys. Commun., 241 (August), 40 (2019). DOI: 10.1016/J.CPC.2019.03.017

[9] А. Бернштейн, Л. Фридленд. Неустойчивости плазмы. Геометрическая оптика нестационарной и неоднородной плазмы в кн. Основы бизики плазмы. Т. 1. Под ред. А.А. Галеев. Р. Судан (Энергоатомиздат, М., 1983), гл. III, c. 393. 